\title{
COMPOSIÇÃO CENTESIMAL DO LEITE HUMANO E CARACTERIZAÇÃO DAS PROPRIEDADES FÍSICO- QUÍMICAS DE SUA GORDURA
}

Roberta Claro da Silva, Jonas Peixoto Escobedo e Luiz Antonio Gioielli*

Departamento de Tecnologia Bioquímico-Farmacêutica, Faculdade de Ciências Farmacêuticas, Universidade de São Paulo, Av. Prof. Lineu Prestes, 580, 05508-900 São Paulo - SP, Brasil

Virgínia Spinola Quintal, Silvia Maria Ibidi e Edna Maria Albuquerque

Departamento de Pediatria, Hospital Universitário, Faculdade de Medicina, Universidade de São Paulo, Av. Prof. Lineu Prestes, 2565, 05508-900 São Paulo - SP, Brasil

Recebido em 29/5/06; aceito em 1/2/07; publicado na web em 30/7/07

\begin{abstract}
CENTESIMAL COMPOSITION OF HUMAN MILK AND PHYSICO-CHEMICAL PROPERTIES OF ITS FAT. Human milk fat is essential for development of newborn infants. Many studies detail chemical characteristics of human milk fat; however there are no studies about its physical properties. The objective of this work was to analyze the centesimal composition of human milk and to compare the calculated energy value with the estimated energy by the creamatocrit method. Chemical composition and physical properties of human milk lipids and Betapol - a structured lipid - were also studied. The results showed that energy values of human milk estimated by creamatocrit and calculated by the centesimal composition didn't present significant correlation. Human milk lipids and Betapol presented distinct physico-chemical properties.
\end{abstract}

Keywords: human milk; creamatocrit; Betapol.

\section{INTRODUÇÃO}

O leite humano é o único alimento energético, nutricional e imunológico consumido em quantidades suficientes pelos recém-nas$\operatorname{cidos}^{1,2}$. O aleitamento materno fortalece a imunidade, mantém o crescimento e desenvolvimento normais, melhora o processo digestivo no sistema gastro-intestinal, favorece o vínculo mãe-filho e facilita o desenvolvimento emocional, cognitivo e do sistema nervoso ${ }^{2-4}$.

O leite humano contém de 3 a $5 \%$ de lipídios, dentre os quais $98 \%$ são de triacilgliceróis, $1 \%$ de fosfolipídios e $0,5 \%$ de esteróis ${ }^{5}$. Os lipídios apresentam-se na forma de glóbulos de cerca de $4 \mu \mathrm{m}$ de diâmetro em emulsão do tipo óleo em água, que é estabilizada por uma membrana contendo fosfolipídios e proteínas ${ }^{6}$. Cerca de $50 \%$ do valor calórico total do leite humano é proveniente da gordura, que é fonte de colesterol, ácidos graxos essenciais e vitaminas lipossolúveis ${ }^{7}$. O conteúdo de ácidos graxos insaturados no leite humano é maior que no de leite de vaca ${ }^{8}$.

As proteínas do leite humano são qualitativamente diferentes das do leite de vaca. Do conteúdo protéico no leite humano, $80 \%$ é lactoalbumina, enquanto que no leite de vaca essa proporção é de caseína. A relação proteínas do soro/caseína no leite humano é aproximadamente 80/20, enquanto a no leite bovino é 20/80. A baixa concentração de caseína no leite humano resulta na formação de coalho gástrico mais leve, com flóculos de mais fácil digestão e com reduzido tempo de esvaziamento gástrico. O leite humano contém também, diferentemente do leite de vaca, maiores concentrações de aminoácidos essenciais de alto valor biológico (cistina e taurina) que são fundamentais ao desenvolvimento do sistema nervoso central. Isso é particularmente importante para o prematuro, que não consegue sintetizálos a partir de outros aminoácidos por deficiência enzimática9 ${ }^{9}$.

A partir da década de 70, com o incentivo ao aleitamento materno, houve crescimento na pesquisa sobre o leite humano ${ }^{10}$. Assim, as nutrizes foram incentivadas a ordenhar e doar o excesso de

*e-mail: lagio@usp.br leite produzido, ficando os bancos de leite humano responsáveis pela atividade de coleta, processamento e controle de qualidade de colostro e leite humano, para posterior distribuição ${ }^{11}$.

$\mathrm{O}$ leite humano ordenhado contém, aproximadamente, $40 \mathrm{~g}$ de gordura/L e $700 \mathrm{kcal} / \mathrm{L}$. Um dos métodos para o controle de qualidade do leite humano é o crematócrito, um micrométodo rápido através do qual se determina a quantidade de creme e se estimam a concentração de gordura e o conteúdo energético de uma amostra $^{12}$. O método de Lucas et al. ${ }^{13}$ é o mais utilizado, sendo recomendado pela Rede Nacional de Bancos de Leite.

Mesmo com todo o esforço, os bancos de leite ainda utilizam fórmulas infantis para suplementação da alimentação de recémnascidos. As fórmulas infantis disponíveis no mercado utilizam em sua composição fontes lipídicas de origem vegetal que têm a estrutura de triacilgliceróis diferente da gordura do leite humano, levando a perdas na absorção de nutrientes. Como as gorduras disponíveis na natureza não possuem tal estrutura, a alternativa seria a utilização de gorduras modificadas por interesterificação.

A empresa Loders \& Croklaan comercializa um sucedâneo da gordura do leite humano que tem o nome comercial BETAPOL ${ }^{\mathrm{TM}} \mathrm{e}$ se baseia na modificação enzimática de tripalmitoilglicerol e óleo de girassol ${ }^{14}$. Shimada et al. ${ }^{15}$ sintetizaram 1,3-araquidonoil-2palmitoil-glicerol pela acidólise da estearina de palma com ácido araquidônico usando a lípase sn-1,3 específica de Rhizopus delemar. No Japão, Akimoto et al. ${ }^{16}$ patentearam um método enzimático similar para produzir sucedâneo da gordura do leite humano rico em ácidos graxos poliinsaturados. Christensen e Holmer ${ }^{17}$, utilizando a enzima sn-1,3 específica de Rhizomucor miehei, conduziram a produção de lipídios estruturados a partir da manteiga obtendo gordura semelhante à do leite humano quanto à composição de ácidos graxos e à sua posição na cadeia dos triacilgliceróis. Os ácidos graxos saturados do leite humano constituem cerca de $41 \%$, sendo predominante o ácido palmítico. Dentre os insaturados, o oléico e o linoléico estão presentes em maior quantidade. Contudo, pouco se conhece sobre as propriedades físicas da gordura do leite humano. 
Este trabalho teve por objetivo analisar a composição centesimal de amostras de leite humano e comparar o valor energético estimado pelo método de crematócrito com o calculado a partir da composição centesimal. Também teve por finalidade caracterizar a gordura do leite humano e um sucedâneo da gordura do leite humano, quanto à composição química e propriedades físicas.

\section{PARTE EXPERIMENTAL}

\section{Amostras}

As atividades referentes à pesquisa com leite humano e a sua manipulação foram aprovadas pelos Comitês de Ética em Pesquisa com Seres Humanos do Hospital Universitário (HU/USP) e da Faculdade de Ciências Farmacêuticas (FCF/USP).

Foram utilizadas amostras de leite materno provenientes de 32 mães em diferentes estágios de lactação, sendo que 19 estavam na fase de colostro e 13 no estágio de leite maduro. As amostras foram doadas pelo Banco de Leite do Hospital Universitário USP-SP.

O leite foi pasteurizado $\left(62,5^{\circ} \mathrm{C}\right.$ por $\left.30 \mathrm{~min}\right)$ e armazenado sob congelamento.

Para a análise das propriedades físicas, a fase gordurosa foi separada de $20 \mathrm{~L}$ de leite em diferentes fases de lactação, utilizando centrífuga contínua de pratos cônicos Alfa-Laval, obtendo-se o creme de leite (emulsão óleo em água). Este, por sua vez, foi transformado em emulsão água em óleo utilizando batedeira planetária. A fase gordurosa da emulsão água em óleo foi separada por fusão à temperatura de $70{ }^{\circ} \mathrm{C}$, seguida por decantação e filtração em papel de filtro. A gordura assim obtida foi armazenada sob congelamento.

O produto Betapol foi gentilmente doado pela empresa Loders Croklaan, divisão de Lipid Nutrition (Holanda).

\section{Métodos}

Os teores de lipídios, umidade, cinzas e proteína foram obtidos pela utilização da metodologia do Instituto Adolfo Lutz ${ }^{18}$, em triplicata. Os carboidratos foram determinados por diferença.

A gordura obtida a partir do leite humano foi analisada quanto à acidez $^{18}$; índice de iodo, calculado a partir da composição em ácidos graxos (método AOCS Cd 1c-85) ${ }^{19}$; diâmetro e número dos cristais, por microscopia sob luz polarizada ${ }^{20-23}$; pontos de fusão e amolecimento $^{19}$ (métodos AOCS Cc 1-25 e Cc 3-25); consistência ${ }^{24}$ e conteúdo de gordura sólida (método AOCS Cd 16-81) ${ }^{19}$. A preparação de ésteres metílicos de ácidos graxos foi realizada seguindo a metodologia de Hartman e Lago ${ }^{25}$. A determinação da composição em ácidos graxos foi realizada em cromatógrafo a gás Varian GC, modelo 3400CX nas condições descritas por Díaz Gamboa e Gioielli ${ }^{24}$.

A análise de crematócrito foi realizada pelo Hospital Universitário da USP, segundo a metodologia indicada pela Rede Nacional de Bancos de Leite ${ }^{12}$. O método do crematócrito consiste na centrifugação do leite humano por $15 \mathrm{~min}$, para a separação do creme e do soro do leite. O creme ocupa a parte posterior do capilar e corresponde à fração de coloração mais densa. O soro, de aspecto menos viscoso, fica abaixo do creme. Com auxílio de uma régua milimetrada mede-se o comprimento da coluna de creme $(\mathrm{mm})$ e da coluna total do produto (coluna de creme + coluna de soro, expressos em mm). De posse destes valores, empregando as fórmulas abaixo, obtém-se:

Teor de creme $(\%)=\frac{\text { coluna de creme }(\mathrm{mm}) \times 100}{\text { coluna total de produto }(\mathrm{mm})}$

Teor de gordura $=\frac{\text { teor de creme }(\%)-0,59}{1,46}$
Energia $(\mathrm{kcal} / \mathrm{L})=66,8 \times$ teor de creme $(\%)+290$

\section{RESULTADOS E DISCUSSÃO}

\section{Composição centesimal}

A composição média obtida para o leite humano nos estágios de colostro e maduro é apresentada na Tabela 1. Os valores obtidos para proteína e carboidratos estão próximos aos citados na literatura $^{26-28}$. Entretanto, a média dos teores lipídicos das amostras apresentou-se abaixo do esperado. Os valores encontrados na literatura indicam teores superiores a $3 \%$ de lipídios totais ${ }^{12,26}$. Esta diminuição pode ter ocorrido devido ao complexo processo: pré-estocagem, congelamento, descongelamento, pasteurização, novo congelamento, novo descongelamento e aquecimento a que o leite foi submetido. Durante todo esse processo são verificadas perdas energéticas significativas, principalmente no teor de gordura, principal fonte calórico-energética do leite humano. Os processos de congelamento e descongelamento, por sua vez, acarretam rompimento das membranas dos glóbulos de gordura, propiciando sua coalescência e facilitando sua aderência às paredes dos frascos armazenadores ${ }^{12,29}$. Assim, o crematócrito medido nas amostras descongeladas pode resultar em baixos valores de lipídios, provavelmente devido à lipólise do leite humano ${ }^{29}$.

Tabela 1. Composição centesimal média de colostro e leite humano maduro

\begin{tabular}{lccc}
\hline Colostro $(\mathrm{n}=19)$ & $\begin{array}{c}\text { Média } \pm \text { desvio } \\
\text { padrão }(\%)\end{array}$ & $\begin{array}{c}\text { Leite maduro } \\
(\mathrm{n}=13)\end{array}$ & $\begin{array}{c}\text { Média } \pm \text { desvio } \\
\text { padrão }(\%)\end{array}$ \\
\hline Umidade & $87,82 \pm 1,09$ & Umidade & $89,85 \pm 0,73$ \\
Carboidratos & $7,80 \pm 0,57$ & Carboidratos & $7,50 \pm 0,66$ \\
Proteínas & $1,85 \pm 0,71$ & Proteínas & $1,12 \pm 0,28$ \\
Lipídios & $1,84 \pm 0,43$ & Lipídios & $1,38 \pm 0,60$ \\
Cinzas & $0,30 \pm 0,16$ & Cinzas & $0,24 \pm 0,09$ \\
\hline
\end{tabular}

O desvio padrão do valor energético das amostras a partir do crematócrito foi maior que a partir da composição centesimal (Tabela 2). A faixa de valor energético estimada pelo crematócrito variou de 40,5 a 97,2 kcal/100 g para o colostro e de 42,1 a 84,6 kcal/100 g para o leite maduro. Por outro lado, o valor energético calculado a partir da composição centesimal variou de 44,8 a 73,1 kcal/100 g para o colostro e de 39,6 a $59,8 \mathrm{kcal} / 100 \mathrm{~g}$ para o leite maduro.

Os valores de energia calculados pelo método do crematócrito e a partir da composição centesimal foram correlacionados separadamente (colostro e maduro). Os resultados mostraram que os valores energéticos estimados pelos dois métodos não apresentaram correlação significativa, embora outros trabalhos da literatura indiquem o contrário ${ }^{13,29,30}$.

Por outro lado, a correlação linear entre o valor energético estimado pelo crematócrito e o teor lipídico obtido na composição centesimal foi significativa, sendo que o $\mathrm{R}^{2}$ foi de 0,57 para o colostro e de 0,75 para o leite maduro. Esta alta correlação também foi encontrada por Wang et al..$^{29}$ e Meier et al. ${ }^{31}$.

As médias dos valores energéticos estimados pelo crematócrito e calculadas a partir da composição centesimal (Tabela 2) estiveram bem próximas para o colostro, e coerentes com os valores citados por Bortolozo et al. $^{28}$, que encontraram média de $57,36 \pm 8,37 \mathrm{kcal} / 100 \mathrm{~g}$, e por Hosoi et al..$^{27}$, que obtiveram média de $57,1 \pm 8 \mathrm{kcal} / 100 \mathrm{~g}$.

Contrariando a literatura ${ }^{32-34}$, neste trabalho foram obtidos maiores valores energéticos no colostro que no leite maduro (Tabela 2), embora os resultados possam ser considerados estatisticamente iguais em função do alto desvio padrão. 
Tabela 2. Média dos valores energéticos estimados pelo crematócrito e calculados pela composição centesimal

\begin{tabular}{|c|c|c|c|}
\hline Colostro $(n=19)$ & $\begin{array}{l}\text { Valor energético } \\
\text { médio } \pm \text { desvio } \\
\text { padrão }(\mathrm{kcal} / 100 \mathrm{~g})\end{array}$ & $\begin{array}{l}\text { Leite maduro } \\
\qquad(\mathrm{n}=13)\end{array}$ & $\begin{array}{l}\text { Valor energético } \\
\text { médio } \pm \text { desvio } \\
\text { padrão }(\mathrm{kcal} / 100 \mathrm{~g})\end{array}$ \\
\hline Crematócrito & $56,60 \pm 13,50$ & Crematócrito & $53,87 \pm 12,52$ \\
\hline Composição centesimal & $56,69 \pm 6,30$ & Composição centesimal & $46,70 \pm 5,63$ \\
\hline
\end{tabular}

Os lipídios são os componentes mais variáveis na composição do leite humano e sua quantidade pode variar em função do período de lactação (colostro e maduro), do período da mamada (diurno ou noturno) e ao longo de cada mamada (leite do início e do fim) ${ }^{5}$.

\section{Propriedades físico-químicas da gordura do leite humano e do Betapol $^{\mathrm{TM}}$}

A acidez da gordura do leite humano apresentou valor relativamente alto, de 2,84 g ácido oléico/100 g amostra, embora não tenham sido encontrados na literatura dados correspondentes. $\mathrm{O}$ tempo entre a coleta das amostras, manipulação e armazenamento são fatores que possivelmente acarretam danos hidrolíticos às amostras, causados possivelmente pela ação de lipases que estão naturalmente presentes no leite humano 5 . O Betapol ${ }^{\mathrm{TM}}$ apresentou acidez de $0,05 \mathrm{~g}$ ácido oléico/ $100 \mathrm{~g}$, valor coerente com as especificações do produto.

Os valores encontrados para a composição em ácidos graxos por cromatografia gasosa (Tabela 3) estão adequados às especificações fornecidas pelo fabricante do Betapol. Em relação ao leite humano, a composição em ácidos graxos situou-se na faixa encontrada na literatura ${ }^{34-36}$, com exceção dos ácidos oléico e linoléico. Mesmo sendo uma gordura exclusivamente vegetal, o Betapol $^{\mathrm{TM}}$ possui $52 \%$ de ácidos graxos saturados, sendo rica nos ácidos láurico, palmítico, oléico e linoléico. Por outro lado, a gordura do leite humano apresentou-se rica nos ácidos palmítico, oléico e linoléico, com $44 \%$ de ácidos graxos saturados.

Tabela 3. Composição em ácidos graxos, acidez e índice de iodo do Betapol ${ }^{\mathrm{TM}}$ e da gordura do leite humano

\begin{tabular}{lcc}
\hline Ácido graxo (\%) & Betapol $^{\mathrm{TM}}$ & Leite humano $^{\circ}$ \\
\hline $10: 0$ & 1,0 & 1,3 \\
$12: 0$ & 17,3 & 7,0 \\
$14: 0$ & 5,7 & 8,0 \\
$16: 0$ & 25,8 & 21,6 \\
$16: 1$ & - & 2,2 \\
$18: 0$ & 2,6 & 6,1 \\
$18: 1 \mathrm{n}-9$ & 35,1 & 27,7 \\
$18: 1 \mathrm{n}-9 \mathrm{t}$ & - & 4,1 \\
$18: 2 \mathrm{n}-6$ & 11,2 & 19,1 \\
$18: 3 \mathrm{n}-3$ & 1,2 & 1,2 \\
$20: 2$ & - & 0,9 \\
$20: 4 \mathrm{n}-6$ & - & 0,6 \\
Acidez (g ácido oléico/100 g) & 0,05 & 2,84 \\
Índice de iodo (g iodo/100 g) & 53 & 60 \\
\hline
\end{tabular}

As médias obtidas para os pontos de fusão e amolecimento da gordura do leite humano foram de 34,8 e $31,5^{\circ} \mathrm{C}$, respectivamente, e encontram-se próximas ao valor descrito pela literatura, que é de $32{ }^{\circ} \mathrm{C}^{34}$. As médias para os pontos de fusão e amolecimento do Betapol $^{\mathrm{TM}}$ foram de 32,1 e $26,9^{\circ} \mathrm{C}$, respectivamente, sendo de 3 a 4 ${ }^{\circ} \mathrm{C}$ abaixo dos valores da gordura do leite humano.

A Figura 1 apresenta a consistência das amostras em diversas temperaturas. $\mathrm{O}$ aumento de temperatura provocou queda brusca na consistência do Betapol $^{\mathrm{TM}} \mathrm{e}$ do leite humano devido à fusão gradual dos cristais e conseqüente destruição da rede cristalina, que confere plasticidade à gordura. Assim, as amostras apresentaram-se mais plásticas a temperaturas entre 15 e $20^{\circ} \mathrm{C}^{37}$.

O comportamento de consistência e conteúdo de gordura sólida (Figura 2) das amostras foi bastante semelhante. Em baixas temperaturas $\left(5\right.$ e $\left.10{ }^{\circ} \mathrm{C}\right)$ o Betapol apresentou-se como uma gordura mais consistente que a do leite humano (Figura 1), o que foi confirmado pelo comportamento da curva de conteúdo de gordura sólida (Figura 2). Com o aumento da temperatura, o Betapol tornou-se uma gordura mais macia que a do leite humano, tendo o conteúdo de gordura sólida acompanhado esse mesmo comportamento.

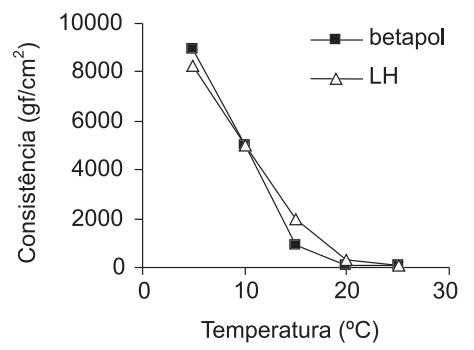

Figura 1. Consistência do Betapol e da gordura do leite humano

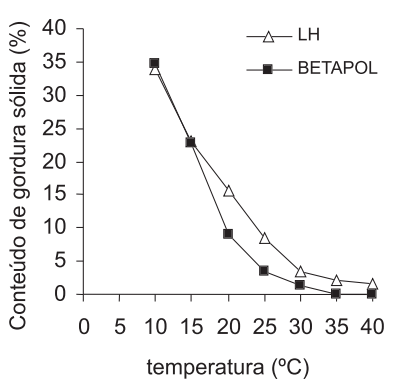

Figura 2. Conteúdo de gordura sólida do Betapol e da gordura do leite humano

O conteúdo de gordura sólida e a consistência apresentaram alta correlação, tanto para a gordura do leite humano como para o Betapol, com $\mathrm{R}^{2}$ de 0,97 e 0,98 , respectivamente.

Quando o conteúdo de gordura sólida é baixo, ao redor de 3 a $5 \%$, a consistência é igual a zero. Isto mostra que quando o teor de sólidos na gordura é muito baixo, o material apresenta-se como um líquido viscoso, ao invés de se comportar como uma gordura plástica. Quando a proporção de gordura no estado sólido é maior que cerca de $5 \%$, o óleo líquido é imobilizado pela matriz cristalina, tornando a gordura plástica ${ }^{38,39}$.

A Tabela 4 apresenta o diâmetro médio e o número de cristais observados por microscopia sob luz polarizada. Como a velocidade de nucleação aumenta exponencialmente com o aumento da supersaturação, enquanto a velocidade de crescimento dos cristais aumenta linearmente com a supersaturação, o número de cristais aumenta e o tamanho dos cristais diminui quando a cristalização ocorre a baixas temperaturas, o que pode ser observado na Tabela $4^{40,41}$. 
Tabela 4. Diâmetro médio e número dos cristais da gordura do leite humano e do Betapol ${ }^{\mathrm{TM}}$

\begin{tabular}{lccccccc}
\hline & \multicolumn{3}{c}{$\begin{array}{c}\text { Gordura do } \\
\text { leite humano }\end{array}$} & \multicolumn{3}{c}{ Betapol $^{\mathrm{TM}}$} \\
\hline & $15^{\circ} \mathrm{C}$ & $20^{\circ} \mathrm{C}$ & $25^{\circ} \mathrm{C}$ & $15^{\circ} \mathrm{C}$ & $20{ }^{\circ} \mathrm{C}$ & $25^{\circ} \mathrm{C}$ \\
Diâmetro médio $(\mu \mathrm{m})$ & 6,3 & 62,1 & 256,0 & 12,4 & 119,2 & 189,0 \\
Numero de cristais & 5496 & 248 & 14 & 1049 & 76 & 6 \\
\hline
\end{tabular}

A Figura 3 apresenta a estrutura cristalina das amostras, por cristalização lenta na faixa de temperatura de 15 a $25{ }^{\circ} \mathrm{C}$. O leite humano e o Betapol ${ }^{\mathrm{TM}}$ apresentaram cristalização homogênea e granular a $15^{\circ} \mathrm{C}$. $\mathrm{O}$ aumento da temperatura produziu aglomerados de cristais a $20^{\circ} \mathrm{C}$, com a formação de esferulitos mais organizados a $25{ }^{\circ} \mathrm{C}$. Estes esferulitos podem ser classificados como do tipo A, pois apresentam cristais com núcleo compacto, cercados de agulhas longas e finas, distribuídas radialmente ${ }^{40}$. Para a gordura do leite humano, os esferulitos podem corresponder à forma polimórfica $\beta$, pois o alto nível de ácido palmítico na posição sn-2 da gordura do leite ${ }^{35,39,42}$ torna os triacilgliceróis com estrutura molecular homogênena. Por outro lado, os esferulitos do Betapol mostraram-se opticamente ativos ("axial crosses"), que possivelmente representam cristalização na forma polimórfica $\beta$-prima, pois o produto Betapol apresenta alto teor de ácido láurico, o que torna os triacilgliceróis com estrutura molecular mais heterogênea.
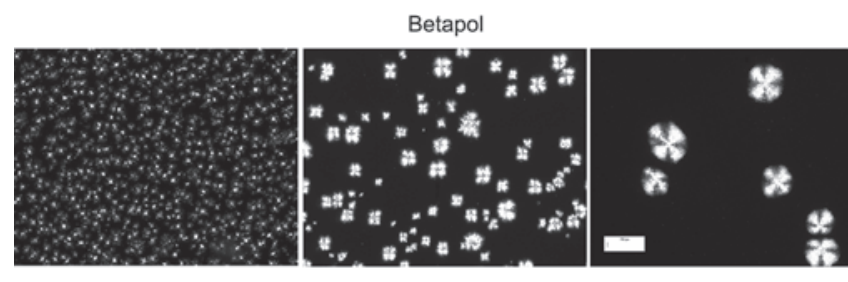

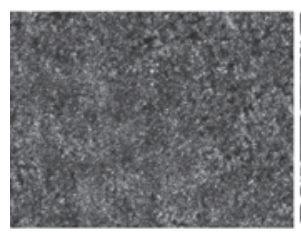

$15^{\circ} \mathrm{C}$
Gordura do leite humano

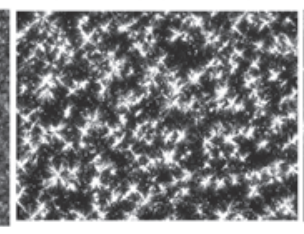

$20^{\circ} \mathrm{C}$

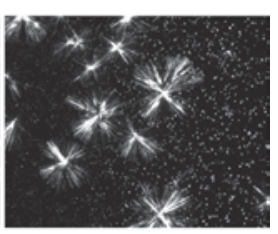

$25^{\circ} \mathrm{C}$
Figura 3. Estrutura cristalina da gordura do leite humano e do Betapol ${ }^{\mathrm{TM}}$. A barra representa $250 \mu \mathrm{m}$

\section{CONCLUSÕES}

Os valores de energia calculados pelo método do crematócrito e a partir da composição centesimal não apresentaram correlação significativa. Por outro lado, a correlação entre o valor energético estimado pelo crematócrito e o teor lipídico foi significativa.

O Betapol apresentou menores consistência, conteúdo de gordura sólida e pontos de fusão e amolecimento que a gordura do leite humano. Os comportamentos de cristalização também foram diferentes.

\section{AGRADECIMENTOS}

À FAPESP, CNPq e CAPES, pelo apoio financeiro e bolsas concedidas aos autores.

\section{REFERÊNCIAS}

1. Almeida, J. A. G.; Amamentação: um híbrido natureza-cultura, Ed. Fiocruz: Rio de Janeiro, 1999.

2. Nobrega, F. J.; Campos, A. L. R.; Distúrbios nutricionais e fraco vínculo mãe/filho, Revinter: Rio de Janeiro, 1996.

3. Anderson, J. W.; Johnstone, B. M.; Ramley, D. T.; Am. J. Clin. Nutr. 1999, $70,525$.

4. Uauy, R.; Mena, P.; Nutr. Rev. 2001, 59, 34.

5. Jensen, R.G.; Lipid Technology 1998, 3, 34.

6. Christie, W. W.; Advanced dairy chemistry - 2 Lipids, Chapman \& Hall: London, 1995.

7. Innis, S. M.; Dyer, R.; Nelson, C. M.; Lipids 1994, 29, 8

8. Silva, M. H. L.; Silva, M. T. C.; Brandão, S. C. C.; Gomes, J. C.; Peternelli, L. A.; Franceschini, S. C. C.; Food Chem. 2005, 93, 297.

9. http://www.aleitamento.org.br/manual/composi.htm, acessada em Março 2006.

10. Wright, A. J.; Hartel, R. W.; Narine, S. S.; Marangoni, A. G.; J. Am. Oil Chem. Soc. 2000, 77, 463.

11. Gibbs, J. H.; Fischer, C.; Bhattacharya, S.; Goddard, P.; Baum, J. D.; Early Hum. Dev. 1977, 1, 227.

12. Cavalcante, J. L. P.; Telles, F. J. S.; Peixoto, M. M. L. V.; Rodrigues, R. C. B.; Ciênc. Tecnol. Alim. 2005, 25, 105.

13. Lucas, A.; Gibbs, J. A. H.; Cyster, R. L. J.; Baun, J. D.; Br. Med. J. 1978, $1,1018$.

14. Quilan, P.; Moore, S.; Inform 1993, 4, 580.

15. Shimada, Y.; Nagao, T.; Hamasaki, Y.; Akimoto, K.; Sugihara, A.; Fujikawa, S.; Komemushi, S.; Tominaga, Y.; J. Am. Oil Chem. Soc. 2000, 77, 89.

16. Akimoto, K.; Yamaguchi, T.; Fujikawa, S.; Europen Patent 0965578 Al, 1999.

17. Christensen, T. C.; Holmer, G.; Milchwissenschaft 1993, 48, 543.

18. Instituto Adolfo Lutz; Normas Analíticas do Instituto Adolfo Lutz, $3^{\mathrm{a}}$ ed., São Paulo, 1985.

19. American Oil Chemists Society; Official methods and recommended practices of the AOCS, $4^{\text {th }}$ ed Champaign, 1990.

20. Simões, I. S.; Gioielli, L.A.; Rev. Bras. Ciênc. Farm. 1999, 35, 259.

21. Simões, I. S.; Gioielli, L. A.; Braz. Arch. Biol. Technol. 2000, 43, 241.

22. Sotero-Solis, V. E.; Gioielli, L. A.; Alimentaria 2001, 38, 131.

23. Gioielli, L. A.; Simões, I. S.; Rodrigues, J. N.; J. Food Eng. 2003, 57, 347.

24. Díaz Gamboa, O. W.; Gioielli, L. A.; Grasas Aceites 2003, 54, 122.

25. Hartman-Lago, R. C. A.; Lab. Pract. 1993, 475, 494.

26. Vieira, A. A.; Moreira, M. E. L.; Rocha, A. D.; Pimenta, H. P.; Lucena, S. L.; J. Pediatr. 2004, 80, 490.

27. Hosoi, S.; Kazuhiro, H.; Daimatsu, T.; Kiyokawa, M.; Aikawa, T.; Watanabe, S.; Pediatrics International 2005, 47, 7.

28. Bortolozo, E. A. F.; Tiboni, E. B.; Candido L. M. B.; Rev. Panam. Salud Publica 2004, 16, 199.

29. Wang, C. D.; Chu, P. S.; Mellen, B. G.; Shenai, J. P.; J. Perinatol. 1999, 10,343 .

30. Collares, F. P.; Gonçalves, C. V.; Ferreira, J. S.; Food Chem. 1997, 60, 465.

31. Meier, P. P.; Engstrom, J. L.; Murtaugh, M. A.; Vasan, U.; Meier, W. A.; Shanler, R. J.; J. Perinatology 2002, 22, 623.

32. Goldfarb, M. F.; Savadone, M. S.; J. Pediatr. Gastroenterol. Nutr. 1991, 12,142 .

33. Hamosh, M.; Bitman, J.; Lipids 1992, 27, 848.

34. Blanc. B.; World Rev. Nutr. Diet. 1981, 36, 1-89.

35. Lien, E. L; Boyle, F. G.; Yuhas, R.; Tomarelli, R. M.; Quinlan, P.; J. Pediatric Gastroentereology \& Nutrition 1997, 25, 167.

36. Christie, W. W.; Clapperton, J. L.; J. Soc. Dairy Tecnol. 1982, 35, 22.

37. Haighton, A. J.; J. Am. Oil Chem. Soc. 1959, 36, 345.

38. Larsson, K.; Lipids: Molecular organization, physical functions and technical applications, The Oil Press; Dundee, 1994, p. 7.

39. Silva, R. C.; Gioielli, L. A.; Rev. Bras. Ciênc. Farm. 2006, 42, 223.

40. Simões, I. S.; Gioielli, L. A.; Óleos Grãos 1999, 8, 20.

41. Timms, R. E. Em Developments in oils and fats; Hamilton, R. J., ed.; Blackie: London, 1995, p. 204.

42. Martin, J. C.; Bougnoux, P.; Antonie, J. M.; Lanson, M.; Couet, C.; Lipids 1993, 28,637 . 